\section{Ads against chimp research criticized}

We at the Federation of American Societies for Experimental Biology object to your publication of advertisements from the Humane Society of the United States that seem to misrepresent the importance of chimpanzees in biomedical research and testing (see, for example, facing page 407 in 22/29 December 2011 issue of Nature).

These advertisements claim that chimpanzees are a poor model for research into human disease because of their physiological and immunological differences. This contention is at odds with the findings of a US Institute of Medicine committee appointed to investigate whether chimpanzees are necessary for biomedical and behavioural research (see go.nature.com/ ruthsl).

The committee, composed of scientific experts and led by a bioethicist, concluded that chimpanzees have been valuable models in the past and that, although "most current use of chimpanzees for biomedical research is unnecessary", they are still needed to conclude research on monoclonal antibodies and possibly in the development of a prophylactic hepatitis $\mathrm{C}$ vaccine.

The committee emphasized that an outright ban on biomedical chimpanzee research would not be appropriate, in part because of new, emerging or re-emerging diseases that cannot be studied in non-chimpanzee models.

Joseph C. LaManna FASEB, Bethesda, Maryland, USA. Joseph-LaManna@faseb.org

\section{Turing: Colossus computer revisited}

George Dyson's history of the Colossus computer (Nature 482, $459-460 ; 2012)$ is somewhat misleading. The development of Colossus owed little to the Bletchley Park 'bombe' devices.
After Bill Tutte developed a statistical strategy to tackle the German Fish cypher, Max Newman proposed a machine to implement it, using two paper tapes. With help from the Telecommunications Research Establishment (later the Royal Radar Establishment) and from Frank Morrell's group at the Post Office Research Station at Dollis Hill, the Robinson machines were built. These were slow but validated the technique. Thomas Flowers' group recommended the use of many more vacuum tubes to improve performance, but they were wrongly considered too unreliable by Bletchley Park.

So Flowers built the first Colossus at Dollis Hill instead of Bletchley Park although, in time, Newman spoke to him about his requirements.

One crucial technique developed by Turing and used by Tutte was that of 'deltaing', or using the differences between characters rather than the characters themselves. If Dyson had pointed out that Tutte used this method to detect the statistical non-randomness of plain text, it would also have helped in understanding how different Fish was from Enigma. Henry Shipley Hexham, UK. h.h.shipley.61@cantab.net

\section{Turing: Brain model still incomplete}

In contemplating whether the brain is a good model for machine intelligence (Nature 482, 462-463; 2012), I believe that Alan Turing's principle that the brain performs computations will continue to hold true. But it seems clear from the state of machine intelligence today that we're missing some basic insight into the language of brain computation.

Advances in genetics, electronics and optics are now enabling us to look into simple brains while they're living and behaving, and to observe the activity of every neuron in real time. This influx of information should eventually help us to make intelligence comprehensible and replicable.

A good approach to interpreting these data is the re-creation of neural processing in simulation, from sensation all the way to behaviour. But once we understand what's going on, it should be possible to create machine intelligence that doesn't rely on reproducing low-level chemical dynamics.

Over the coming decades we are likely to take high-level cues from biology on how to organize our silicon. Mind is merely a function of the brain, however, so we should be able to capture that function in any Turingcomplete system once we know how the brain is organized to perform it.

David Dalrymple Cambridge, Massachusetts, USA. davidad@alum.mit.edu

\section{Turing: Beyond the original concept}

Barry Cooper asks whether information can be increased through computation, pointing out that Turing computation does not create anything not already in the initial data (Nature 482, $465 ; 2012)$. If we are limited to Turing machines, then I believe the answer to his question is no. But if we enhance them then information can accumulate.

Turing machines are designed to model functions, not ongoing computations involving additional input over time. But if we enhance Turing machines by giving them a persistent memory and allowing them to alter their input by interacting with their environment, then information can increase.

Interactive machines engage in input and output during computation, which is closer to how computers are used in practice than in the Turing machine framework: examples include operating systems, interactive agents in artificial intelligence, and solutions to some problems in control theory.

This observation is largely unappreciated in theoretical computer science, but a handful of researchers are exploring this part of 'super-Turing' space. Christopher Kanan University of California San Diego, La Jolla, California, USA. ckanan@cs.ucsd.edu

\section{Be sparing with international laws}

We believe that Devi Sridhar's justification for proposing a Framework Convention on Alcohol Control is problematic (Nature 482, 302; 2012). Standard-setting international laws are largely dictated by powerful states, based on expectations that they themselves already meet, obliging poorer states to implement these 'enlightened' global policies ahead of local priorities. Litigation by foreign non-governmental organizations (NGOs) can also get in the way of national policy-making, particularly as most NGOs are led from the West.

Sridhar's proposal joins growing calls for new international health laws. But first we need more evidence that international laws achieve results commensurate with the cost of drafting, ratifying and implementing them.

In our view, some clear criteria need to be fulfilled before the WHO invokes its law-making authority. These could be set by a commission on global health law. Ill-justified international health laws that dictate poor countries' policies and priorities from afar could prevent serious consideration of initiatives better suited to legal instruments.

Steven J. Hoffman McMaster University, Hamilton, Canada. hoffmans@mcmaster.ca John-Arne Røttingen Harvard Kennedy School, Cambridge, Massachusetts, USA. 